\title{
Evaluation of Endometrial Endometrioid Carcinoma Grade using Ultraviolet- Visible-Light Microscopic Spectroscopy
}

Haruhiko Yoshioka $^{1^{*}}$, Hironori Kondo ${ }^{2}$, Kayo Horie ${ }^{1}$, Kiyotada Washiya ${ }^{1}$ and Jun Watanabe ${ }^{1}$

${ }^{1}$ Hirosaki University Graduate School of Health Sciences, Hirosaki, Japan

2/waki Kyoritsu General Hospital, Japan

"Corresponding author: Haruhiko Yoshioka, Hirosaki University Graduate School of Health Sciences, 66-1 Honchou. Hirosaki, Aomori 036-8564, Japan, Tel: +81 172 39 5972; E-mail: yoshioka@hirosaki-u.ac.jp

Received date: Oct 22, 2015; Accepted date: Nov 05, 2015; Published date: Nov 07, 2015

Copyright: $\odot 2015$ Yoshioka H, et al. This is an open-access article distributed under the terms of the Creative Commons Attribution License, which permits unrestricted use, distribution, and reproduction in any medium, provided the original author and source are credited.

\section{Abstract}

Objective: The objective of this study was to investigate the possibility of an objective index to evaluate the grades of endometrial endometrioid carcinomas by analyzing the spectral values of ultraviolet(UV) and visible(Vis) transmittances, and the degree of UV- absorbing materials influencing nuclear colors using ultraviolet- visible-light microscopic spectroscopy (UV-Vis MS).

Study design: The materials were histological preparations from 4 patients, each with proliferative-phase endometrium (PPE) and grade $1(\mathrm{G} 1)$ and grade 3 (G3) of endometrial endometrioid carcinomas. Both spectra were measured in the same 50 nuclei of each specimen.

Results: 1) In the ultraviolet spectrum, transmittance of 260, 280, 300, and $320 \mathrm{~nm}$ were decreased in G3 compared to those in G1. All of the ultraviolet absorbing materials increased in G3. 2) In the visible spectrum, 520 $\mathrm{nm}$ (purplish red), $540 \mathrm{~nm}$ (purple), and $560 \mathrm{~nm}$ (violet) in G3 were deeper than all colors in G1, and violet in G3 was lighter than purplish red and purple in G1. 3) The following 3 characteristics were clarified with regard to the relationship between UV-absorbing materials and the violet color based group. 1) In G3, the number of the significant canonical correlation coefficient between ultraviolet light-absorbing materials and the violet color based group was only at one. 2) Purplish red and purple of the nuclei in G3 was strongly influenced by ultraviolet lightabsorbing materials $(260-320 \mathrm{~nm})$. 3) Purple and violet in $\mathrm{G} 1$ was strongly influenced by light-absorbing materials at $300 \mathrm{~nm}$ and $320 \mathrm{~nm}$.

Conclusion: It was indicated that UV-Vis MS analysis could be an objective index to evaluate the grade of endometrial endometrioid cancerby analyzing the spectral values of transmittances of ultraviolet and visible light, and the degree of ultraviolet light- absorbing materials influencing nuclear colors in the same nuclear region.

Keywords: Endometrial endometrioid adenocarcinoma; Differentiation; Ultraviolet light; Visible light; Microscopic spectroscopy; Unstained tissue preparation; Canonical correlation analysis

\section{Introduction}

The prevalence of endometrial cancer is increasing in Japan due to the westernization of the Japanese lifestyle [1]. Endometrial cancer arises from the uterine endometrium, and is divided into estrogendependent type I and - independent type II. Endometrioid carcinoma accounts for most type-I cases. Endometrioid carcinoma develops from endometrial hyperplasia as a precursor lesion or often accompanies as a background lesion, and shows a broad range of histology from Grade $1(\mathrm{G} 1)$ to grade 3 (G3). G3 often deeply invades the muscle layer and the prognosis is poor. Grading is classified based on structural atypia (partially cellular atypia), but an objective index of differentiation or malignancy other than structural atypia has not been investigated sufficiently. A new objective index to evaluate nuclear atypia is necessary.
Ultraviolet light (UV) is a wavelength from $10 \mathrm{~nm}$ to $380 \mathrm{~nm}$, which is shorter than that of visible light. $260 \mathrm{~nm}$ absorbance is the maximum absorption wavelength of the conjugated double bands in the bases constituting the nucleic acid, and this is known to be important for the quantitation of nucleic acid. $280 \mathrm{~nm}$ absorbance is the maximum absorption wavelength of the aromatic amino acids constituting the proteins, and this is known to be important for the quantitation of proteins [2-5].

The colors of the nucleus are composed of a light mix of various visible wavelengths. The colors of hematoxylin- eosin (H-E) staining include 520, 540, and $560 \mathrm{~nm}$ as complementary color wavelengths of purplish red, purple, and violet, respectively. Therefore, these wavelengths are known to be important for color quantitation [5-9].

Reportedly, tumor development and progression markedly disturb the control of proliferation and differentiation, and abnormalities in histone-modifying enzymes and nuclear matrix protein disturb elements of the intranuclear microenvironment, having an effect on phenotypic changes in the nuclear morphology [10].

One of the important decision items of nuclear grade is nuclear color [11]. However, it is sometimes difficult to distinguish cancer 
nuclear colors with increased euchromatin from that of non-cancer cells because both are similarly clear, and also cancer nuclear colors with increased heterochromatin from that of non-cancer cells with regeneration because both are dark [12].

In this study, we focused on the influence of the substance, in particular, the nucleic acid in $260 \mathrm{~nm}$ and proteins in $280 \mathrm{~nm}$, on the color change of the cancer cell nucleus. No studies have examined the influence of UV-absorbing substances on nuclear color.

The objective of this study was to investigate an objective index to evaluate the grade of endometrioid carcinomas by analyzing the spectral values of ultraviolet and visible transmittances and degree of ultraviolet light- absorbing materials influencing nuclear colors using UV-Vis MS

\section{Materials and Methods}

\section{Patients}

The tissue samples for UV-Vis MS were histological preparations from 4 patients each with proliferative-phase endometrium (PPE), G1 and G3 endometrioid carcinomas. Both spectra were measured in the same 50 nuclei in each sample. The significant differences were analyzed by the Mann-Whitney U-test. The relationship between ultraviolet light-absorbing materials and hematoxylin staining was analyzed using a canonical correlation analysis (CCA). This study was approved by the Hirosaki University School of Medicine and Hospital Ethics Committee (2013-232).

UV-MS samples: The materials for UV- MS were unstained histological preparations. Excised specimens were fixed in 20\% neutral buffered formalin solution and paraffin-embedded, and $3-\mu \mathrm{m}$-thin sections were prepared and mounted on quartz slide glasses (Technical). The preparations were deparaffinized with xylene and alcohol, rinsed with water, dripped with glycerin (Carl Zeiss) without staining, sealed with quartz cover glasses (Technical), and subjected to ultraviolet spectroscopy.

Vis MS samples: For the materials for Vis-MS, the above preparations used for UV-MS were stained with HE. After UV-MS analysis, the quartz cover glasses were removed, and the preparations were rinsed with water. All preparations were simultaneously stained with $\mathrm{HE}$ and sealed with standard cover glasses, and subjected to visible spectroscopy.

\section{Lambert-Beer law in UV-Vis MS}

Light absorption, absorption spectrometry [13], and fluorescent analysis [14] using UV-Vis MS in the analytical chemistry field were applied to histological preparations. Data were collected following the principle of light absorption in analytical chemistry, the Lambert-Beer law [13].

The principle of absorbance measurement is measurement of the amount of light absorbed when monochromatic light passes through a sample. A sample at a concentration of $\mathrm{c}(\mathrm{c} \mathrm{mM})$ is added to an absorption cell. When the intensity of monochromatic light with an intensity of I0 becomes I after passing through the cell with an optical path length, $1, I / I$ 。 represents transmittance and is designated as $\mathrm{T}$. When this is presented as a percentage ratio (\%), it is termed percent transmittance and designated as $\mathrm{T} \%$. $-\log I / I_{\mathrm{o}}$ is termed absorbance and designated as A. Accordingly, absorbance is formulated as:

$$
A=-\log T=-\log \frac{I}{I_{0}}=\varepsilon c l .
$$

$\varepsilon$ represents the extinction coefficient. Absorbance is proportionate to the passage of distance of the light and the concentration of the solution (Lambert-Beer law).

Excluding a few exceptions, absorbance is measured for the total concentration of different chemical species because of the additivity of absorbance. The absorbance of a sample containing $\mathrm{n}$ active absorbance values is presented by the equation below:

$$
A=-\log \frac{I}{I_{0}}=d l \sum_{i=1}^{n} \varepsilon_{i} c_{i}
$$

Using multifunctional software for spectroscopy (Ocean Photonics, Japan), percent transmittance, T\%, was measured on the assumption that this equation is established.

\section{UV-Vis MS apparatus}

The UV-Vis MS was performed using a UV-Vis MS apparatus originally developed by Hirosaki University Graduate School of Health Sciences, and the light source and spectroscope were defined $[4,5]$. For the intermediate filter of the UV-MS light source, a UV-mirror module was set, through which only ultraviolet light $(250-370 \mathrm{~nm})$ can be selected from the broad continuous wavelength range of the light source lamp. For the intermediate filter of the light source of Vis-MS, a Vis-mirror module was set, through which only visible light (400-700 $\mathrm{nm}$ ) can be selected from the broad continuous wavelength range of the light source lamp. Since a high-performance cold mirror is used for the UV-Vis mirror module, unnecessary heating can be completely inhibited, resulting in an extremely low level of stray light. The nuclear measurement area to acquire a spectrum was set at $166.4 \mu \mathrm{m}^{2}(12.9 \times$ $12.9 \mu \mathrm{m})$ in all measured cells. This area was mostly fitted into the nucleus $[4,5]$.

\section{Acquired wavelength and image data}

The ultraviolet transmittance spectrum of $260,280,280,300$, and $320 \mathrm{~nm}$ were extracted by UV-MS. The Visible transmittance spectrum of 520 (purplish red), 540 (purple), and $560 \mathrm{~nm}$ (violet) were extracted by Vis-MS. UV images of UV-MS (Figures 1A and 1C) and Vis images of Vis-MS (Figures 1B and 1D) were acquired using image acquisition software (Basico, CLARO, Japan) in Bitmap format.

To control calibration of the transmittance measurement, the following procedure was performed for each sample. Measurements of 0 and $100 \%$ transmittance control areas were set at sites very close to the sample cell, which eliminated the transmittance of substances around the sample cell and enabled setting the 100\% transmittance baseline.

\section{Statistical analysis}

The UV and Vis-spectral values were determined, and the significant differences were analyzed using the Mann-Whitney U-test. For degree of Ultraviolet light- absorbing materials influencing nuclear colors, the relationship between ultraviolet light-absorbing materials and hematoxylin staining was analyzed using a canonical correlation analysis [15]. 
Citation: Yoshioka H, Kondo H, Horie K, Washiya K, Watanabe J (2015) Evaluation of Endometrial Endometrioid Carcinoma Grade using Ultraviolet- Visible-Light Microscopic Spectroscopy. J Cytol Histol 6: 381. doi:10.4172/2157-7099.1000381

Page 3 of 7

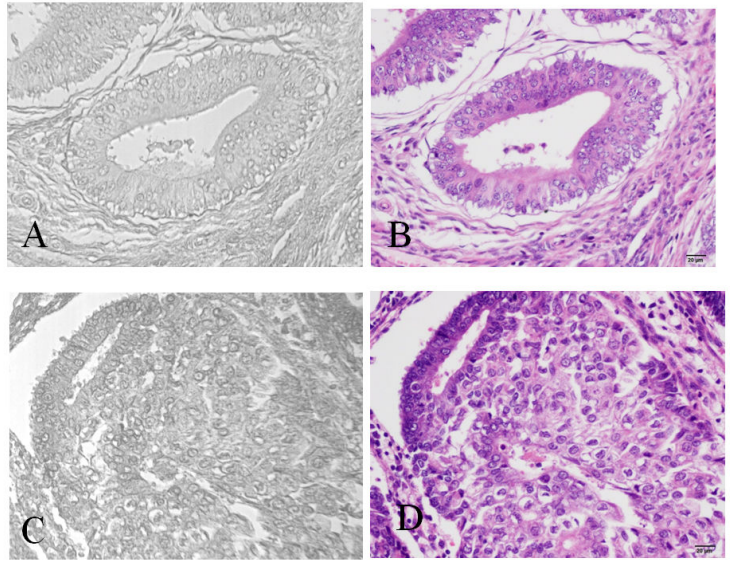

Figure 1: Comparison of UV-MS (A, C), Vis-MS (B, D) imaging, 20X (A) UV absorption image of G1, (B) HE stained image of G1, (3) UV absorption image of G3, (D) HE stained image of G3.

\section{Results}

\section{UV-Vis MS spectral analysis}

UV-MS spectral analysis: Figure 2 shows the ultraviolet spectra of PPE, G1, and G3.

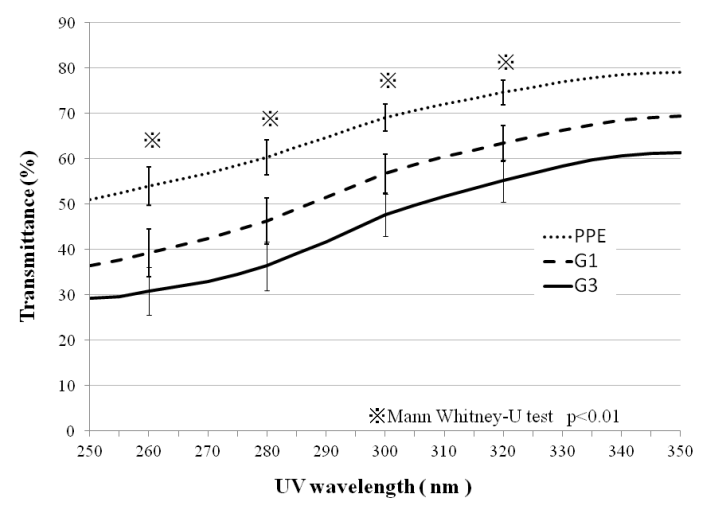

Figure 2: UV spectra of a proliferative-phase endometrium (PPE) and endometrioid adenocarcinoma (G1 and G3). Fine dotted, Coarse dotted and Solid lines indicate transmittance of PPE, G1 and G3, respectively.

The biochemical maximum absorption wavelength of nucleic acids, $260 \mathrm{~nm}$, and of aromatic amino acids, $280 \mathrm{~nm}$, and the absorption wavelength of 300 and $320 \mathrm{~nm}$ were extracted and designated an ultraviolet wavelength group.
The ultraviolet transmittances of G1 and G3 were significantly lower than that of PPE, and that of G3 was significantly lower than that of G1 at all wavelengths.

Vis- MS spectral analysis: Figure 3 shows the visible spectra of PPE, G1, and G3. The visible wavelength of about $450-490 \mathrm{~nm}$ showed a yellow to red color, and this wavelength area is the color area of the eosin pigment. The visible wavelength of about $520-560 \mathrm{~nm}$ showed a purplish red to bluish purple color, and this is the color area of the hematoxylin pigment.

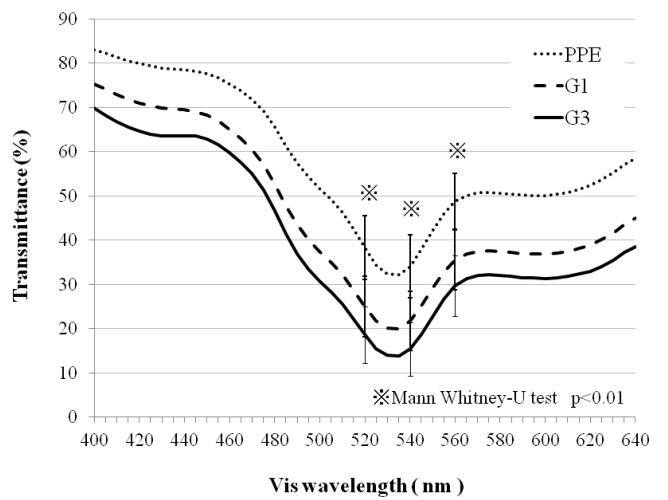

Figure 3: Vis spectra of a proliferative-phase endometrium (PPE) and endometrioid adenocarcinoma (G1 and G3) Fine dotted, Coarse dotted and Solid lines indicate transmittance of PPE, G1 and $\mathrm{G} 3$, respectively.

Wavelengths of 520 (purplish red) 540 (purple), and $560 \mathrm{~nm}$ (violet) were extracted as analytical variates of the hematoxylin color and designated a Violet color based group. The color area of the eosin pigment was excluded from the data to eliminate contamination with cellular components within the nuclear measurement range and artifact elements of excess eosin staining. A low transmittance (\%) indicates high intranuclear absorption of the wavelength (absorption color). The complementary color is perceived as deep as the absorption color increases.

In all of PPE, G1, and G3, the transmittance of the violet color based group started to decrease at about $450 \mathrm{~nm}$, reaching its lowest at about $530 \mathrm{~nm}$, then rising up to $560 \mathrm{~nm}$, when the slope became flat and rose again over the region to $640 \mathrm{~nm}$.

When these changes with wavelength were presented as colors, the nuclear color was deeper in the order of G3> G1> PPE, and the color was deeper in the order of purple>purplish red $>$ violet.

The values at 520 (purplish red) and 540 (purple) nm in G3 were significantly lower than those at all wavelengths in G1. The value at 560 $\mathrm{nm}$ (violet) in G3 was higher than those at 520 (purplish red) and 540 (purple) $\mathrm{nm}$, but lower than that at $560 \mathrm{~nm}$ (violet) in G1. 
Citation: Yoshioka H, Kondo H, Horie K, Washiya K, Watanabe J (2015) Evaluation of Endometrial Endometrioid Carcinoma Grade using Ultraviolet- Visible-Light Microscopic Spectroscopy. J Cytol Histol 6: 381. doi:10.4172/2157-7099.1000381

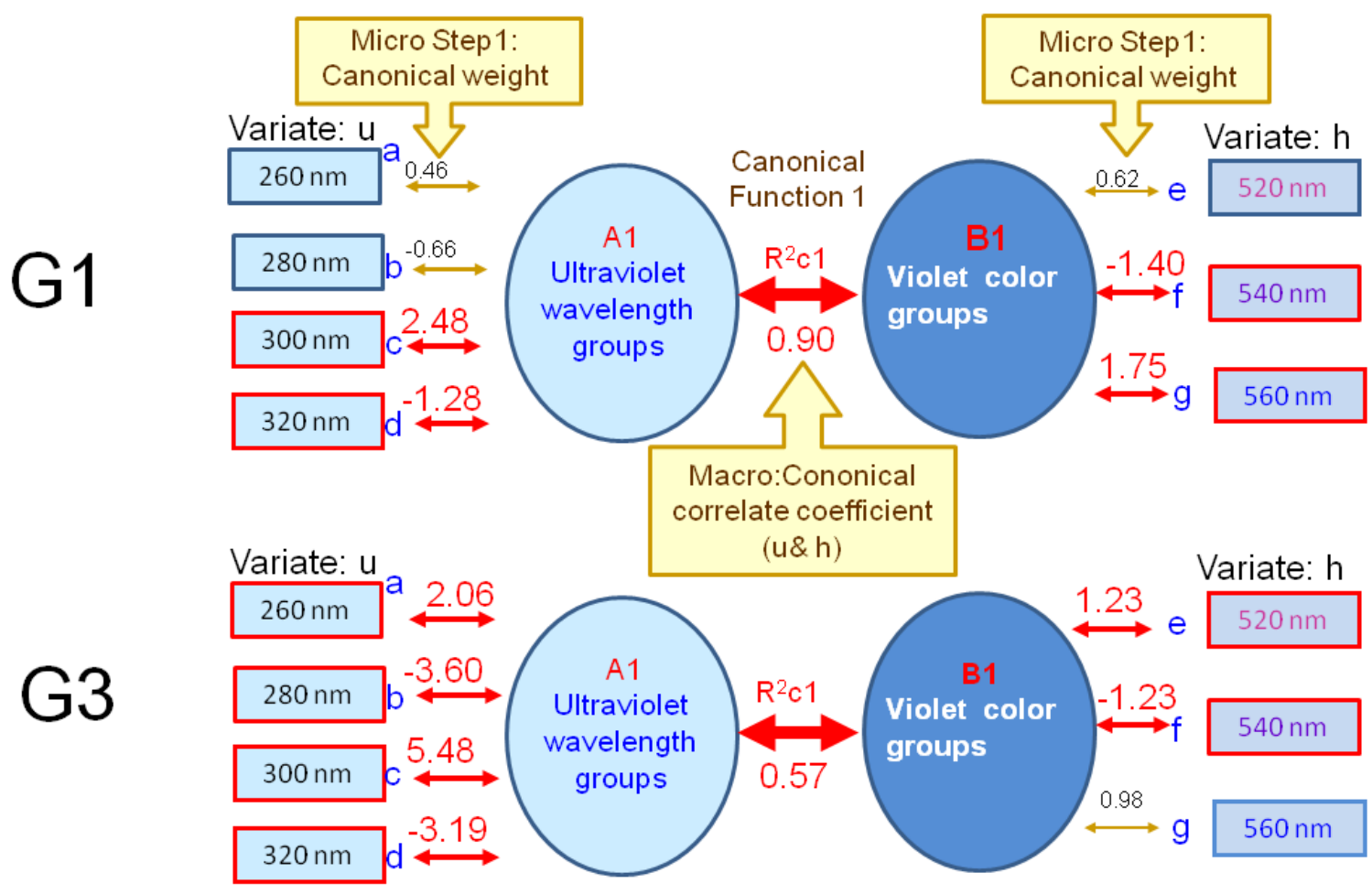

Figure 4: Graphical presentation comparing the standardized canonical coefficients of the first canonical variables in G1 and G3. The standard canonical coefficients are a correlation coefficient that represent the levels of involvement levels of sub-variates of the standardized new variates, $\mathrm{u}$ and $\mathrm{h}$, when $\mathrm{u}$ and $\mathrm{h}$ are most strongly correlated. The canonical variables strongly influence each other as the standard canonical coefficient weight increases. The results of the standard canonical coefficients were analyzed based on the interpretation of the nuclear color.

\section{Degree of Ultraviolet light- absorbing materials influencing nuclear colors}

The relationship between ultraviolet light-absorbing materials and hematoxylin staining in G1 and G3 was analyzed using a canonical correlation analysis (Figure 4) [15].

Design of canonical correlation analysis: To investigate the degree of ultraviolet light-absorbing materials influencing the nuclear color of endometrial cancers, a canonical correlation analysis of the ultraviolet wavelength and violet color based groups was designed. For the variate groups for comparison, the ultraviolet wavelength and violet color based groups were designated as Groups A and B, respectively. The linear model of a new variate, $\mathrm{u}$, of Group A included 4 variates: the transmittance values at 260,280,300, and $320 \mathrm{~nm}$. The linear model of a new variate, $h$, of Group B included 3 variates: the transmittance values at 520,540 , and $560 \mathrm{~nm}$.

Group A: Ultraviolet wavelength group (the new variate was designated as $\mathrm{u}$ )

$\mathrm{u}=\mathrm{a}[260 \mathrm{~nm}]+\mathrm{b}[280 \mathrm{~nm}]+\mathrm{c}[300 \mathrm{~nm}]+\mathrm{d}[320 \mathrm{~nm}]$

Constants: a, b, c, and d
Group B: Violet color based group (the new variate was designated as h)

$$
h=e[520 n m]+f[540 \mathrm{~nm}]+g[560 \mathrm{~nm}]
$$

Constants: e, f, and g

Canonical correlation analysis of the above 2 variate groups was performed [15]. as follows:

The results of the canonical correlation analysis were interpreted

1. Canonical correlation coefficient: Maximum correlation coefficient, $\lambda$, between the new variates, $\mathrm{u}$ and $\mathrm{h}$ (several eigenvectors were present). The new variates, $u$ and $h$, at this coefficient were termed canonical variables. The canonical variable of the best eigenvector (eigenvalue) was designated first canonical function.

2. Standardized canonical coefficients weight: The constant when the new variates, $u$ and $h$, were most strongly correlated. The more standardized canonical coefficients weight is large, the more strongly it will affect the canonical variable.

Significant difference test of canonical variables in canonical correlation analysis: Table 1 shows the results of the significance test of canonical variables in the canonical correlation analysis. In G1, the 
canonical correlation coefficients of the first $(0.899)$ and second $(0.392)$ canonical variables were significant $(\mathrm{P}<0.05)$. In $\mathrm{G} 3$, only the correlation coefficient of the first canonical variable $(0.574)$ was significant $(\mathrm{P}<0.05)$. Thus, the canonical correlation analysis of the first canonical variable proceeded in both G1 and G3.

\begin{tabular}{|l|l|l|l|l|l|l|}
\hline \multirow{2}{*}{$\begin{array}{l}\text { Canonical } \\
\text { Function }\end{array}$} & \multicolumn{6}{|l|}{ G1 } \\
\cline { 2 - 7 } & $\begin{array}{l}\text { Eigen } \\
\text { value }\end{array}$ & $\begin{array}{l}\text { Canonical } \\
\text { correlation }\end{array}$ & $\begin{array}{l}\text { p- } \\
\text { value }\end{array}$ & $\begin{array}{l}\text { Eigen } \\
\text { value }\end{array}$ & $\begin{array}{l}\text { Canonical } \\
\text { correlation }\end{array}$ & $\begin{array}{l}\text { p- } \\
\text { value }\end{array}$ \\
\hline 1 & 0.808 & 0.899 & $<0.01$ & 0.330 & 0.574 & $<0.01$ \\
\hline 2 & 0.154 & 0.392 & $<0.01$ & 0.029 & 0.169 & 0.15 \\
\hline 3 & 0.016 & 0.128 & 0.20 & 0.020 & 0.140 & 0.14 \\
\hline
\end{tabular}

Table 1: Significance of canonical variables in canonical correlation analysis.

Standardized canonical coefficients weights of the first canonical variable in the canonical correlation analysis: Figure 4 shows the results of the standard canonical coefficients of the first canonical variables in G1 and G3. The standard canonical coefficients are the correlation coefficients that represent the involvement levels of subvariates of the standardized new variates, $\mathrm{u}$ and $\mathrm{h}$, when $\mathrm{u}$ and $\mathrm{h}$ are most strongly correlated. The canonical variables strongly influence each other as the standard canonical coefficient weight increases. The results of the standard canonical coefficients were analyzed based on the interpretation of the nuclear color.

Table 2 and Figure 4 show the degree of ultraviolet light- absorbing materials influencing nuclear colors.

\begin{tabular}{|c|c|c|}
\hline \multicolumn{3}{|c|}{$\begin{array}{l}\text { U: Standardized canonical coefficients for the Ultraviolet wavelength } \\
\text { groups }\end{array}$} \\
\hline \multirow[t]{2}{*}{ UV wavelength } & \multicolumn{2}{|c|}{ canonical weights } \\
\hline & G1 & G3 \\
\hline $260 \mathrm{~nm}$ & 0.46 & 2.06 \\
\hline $280 \mathrm{~nm}$ & -0.66 & -3.60 \\
\hline $300 \mathrm{~nm}$ & 2.48 & 5.48 \\
\hline $320 \mathrm{~nm}$ & -1.28 & -3.19 \\
\hline \multicolumn{3}{|c|}{ h: Standardized canonical coefficients for the Violet color groups } \\
\hline \multirow[t]{2}{*}{ Vis wavelength } & \multicolumn{2}{|c|}{ canonical weights } \\
\hline & G1 & G3 \\
\hline $520 \mathrm{~nm}$ & 0.62 & 1.23 \\
\hline $540 \mathrm{~nm}$ & -1.40 & -1.23 \\
\hline $560 \mathrm{~nm}$ & 1.75 & 0.98 \\
\hline
\end{tabular}

Table 2: Comparison of the standardized canonical coefficient of the first canonical variable.

In G1, when the canonical variables were most strongly correlated (first canonical correlation coefficient: 0.899 ) between the A1 and B1 groups, the strongly influencing variates were $300 \mathrm{~nm}$ (standard canonical coefficients: 2.48$)$ and $320 \mathrm{~nm}(-1.28)$ for purple $540 \mathrm{~nm}$
$(-1.40)$ and bluish purple $560 \mathrm{~nm}(1.75)$. The variate, $260 \mathrm{~nm}$, had no strong influence.

In G3, when the canonical variables were most strongly correlated (first canonical correlation coefficient: 0.573 ) between the 2 groups, the strongly influencing variates were $260 \mathrm{~nm}$ (standard canonical coefficients: 2.06), $280 \mathrm{~nm}(-3.60), 300 \mathrm{~nm}$ (5.47), and $320 \mathrm{~nm}(-3.19)$ for purplish red $520 \mathrm{~nm}$ (1.23) and purple $540 \mathrm{~nm}(-1.23)$.

The degree of influence of ultraviolet absorption materials on the changes in the nuclear color in G1 and G3 was different. In G1, ultraviolet absorbing materials influencing the nuclear color variation of purple and violet, received a strong influence from ultraviolet absorbers at $300 \mathrm{~nm}$ and $320 \mathrm{~nm}$. In G3, ultraviolet absorbing materials influencing the nuclear color variation of purplish purple and purple, received a strong influence from ultraviolet absorbers at $260,280,300$, and $320 \mathrm{~nm}$.

\section{Discussion}

\section{Characteristics of ultraviolet spectra of G1 and G3}

In the spectra of the ultraviolet wavelength groups in PPE, G1 and $\mathrm{G} 3$, the ultraviolet transmittance was significantly lower in the order of G3, G1, and PPE at all wavelengths.

Zeskind et al. [2], Cheung et al. [3] observed the nuclear mitosis and motility of viable cells in culture as an absorption image of ultraviolet 260 and $280 \mathrm{~nm}$, and demonstrated nucleotides and protein. Biochemically, nucleotide bases [adenine $(260 \mathrm{~nm})$, guanine $(253 \mathrm{~nm})$, cytosine $(271 \mathrm{~nm})$, and thymine $(267 \mathrm{~nm})]$ and amino acids [tryptophan $(278 \mathrm{~nm})$, tyrosine $(274.5 \mathrm{~nm})$, and phenylalanine $(257.5$ $\mathrm{nm})]$ are considered to be the absorbing materials of 260 and $280 \mathrm{~nm}$ ultraviolet. In addition, substances containing $\mathrm{N}=\mathrm{O}$ and $\mathrm{C}=\mathrm{O}$ absorb wavelengths near $300 \mathrm{~nm}$ and substances containing $-\mathrm{N}=\mathrm{N}$ absorb wavelengths near $350 \mathrm{~nm}$. Nucleic acids such as DNA and RNA, and proteins such as nuclear matrix protein and heterochromatin, increase in cancer cells compared to those in normal cells [10].

In this study it was revealed that the ultraviolet spectral absorption was stronger in the order of G3, G1, and PPE, suggesting that absorbing materials, such as nucleic acids and proteins, increased more in this order.

\section{Nuclear color characteristics of G1 and G3}

In Vis-MS, the violet color group (purplish red at $520 \mathrm{~nm}$, purple at $540 \mathrm{~nm}$, violet at $560 \mathrm{~nm}$ ) in the nuclear color was analyzed.

All violet colors in the nuclear region were deeper in G1 and G3 than in PPE, and the color was deeper in the order of purple, purplish red, and violet. The valley shape of the spectrum was similar between the normal and the cancer cells, and no predominance of a specific color was noted. A predominance of a specific color suggests inappropriate conditions, such as contamination with an artifact, inadequate staining, thick sections, and wrinkles.

Regarding each of the hematoxylin colors in the nuclear region: purplish red, purple, and violet, purplish red and purple in G3 were deeper than all colors in G1, and violet in G3 was lighter than purplish red and purple and deeper than violet in G1. Violet in G1 was lighter than all colors in G3.

It was found that Vis spectral analysis of nuclear color could objectively evaluate the nuclear colors of G1 and G3 at a more detailed 
difference. There is no reports that have studied the spectral difference of nuclear color.

Intranuclear DNA strongly binds to histone protein present in the same amount and forms nucleosomes. Chromosomes contain histone (basic amino acids account for more than 1/5) and non-histone proteins in an equivalent amount, and chromatin comprises a repeated structure of this DNA-protein particle. Chromatin construction involves many amino acids constituting ATP-dependent chromatin remodeling complexes and various enzymes involved in DNA replication and synthesis, and RNA transcription [16].

Histological colors of $\mathrm{H}-\mathrm{E}$ staining are defined by the electric charge state of these various intranuclear materials [6]. The nuclear colors of G3, such as deeper purplish red and purple than in G1 and lighter bluish purple than purplish red and purple in G1, may be characterized due to increases in not only basophilic DNA but also various acidic proteins and enzymes (purplish red). To diagnose the nuclear color of G3, it may be important to pay attention to increases in the purple and purplish red colors and observe pale bluish purple in G3 to differentiate G3 from G1.

\section{The degree of influence of ultraviolet absorption materials on the changes in the nuclear color in G1 and G3}

The nuclear color of H-E staining depends on the electric charge state of intranuclear materials [6]. Thus, nuclear colors are similar when the electric charge state of the nucleus is similar. But, it is not to say that nuclear components are similar when the nuclear colors are similar. In this study, using UV-Vis MS methods that can simultaneously measure the nuclear colors and nuclear ultraviolet absorption material in the same nucleus, the influence degree of ultraviolet absorption material on the nuclear color was investigated. This approach is not affected by any bias of the nuclear staining properties.

The influence degree was statistically analyzed by the canonical correlation analysis to determine the correlation coefficient between the UV spectrum and Vis spectrum change.

The following 3 characteristics were clarified by the analysis.

1) In G3, the number of the significant canonical correlation coefficient between ultraviolet light-absorbing materials and the violet color based group was only at one. The canonical correlation coefficient between ultraviolet light-absorbing materials and the violet color based group was weak in G3 (0.573). This means that changes in the nucleus color of G3 were not due to specifically corresponding ultraviolet absorbing materials.

2) Purplish red and purple of the nuclei in G3 was strongly influenced by ultraviolet light-absorbing materials $(260-320 \mathrm{~nm})$.

3) Purple and violet in G1 was strongly influenced by lightabsorbing materials of $300 \mathrm{~nm}$ and $320 \mathrm{~nm}$. To our knowledge, these 3 characteristics have not previously been reported.

Reportedly, tumor development and progression markedly disturb the control of proliferation and differentiation, and abnormalities of histone-modifying enzymes and nuclear matrix protein disturb elements of the intranuclear microenvironment, having an effect on phenotypic changes in the nuclear morphology [10].

In this study, not only the UV and Vis spectral transmission values, but also the correlation of the UV and Vis transmittance value were different between G1 and G3. It was indicated that various gene expression-regulating proteins and enzymes, and materials absorbing ultraviolet light are markedly more abnormal and disorderly in G3 than compared to those in G1, which may weaken and disrupt the relationship between the hematoxylin stainability and ultraviolet wavelength, and be related to the diversity of intranuclear structures and 3-dimensional changes.

\section{Conclusion}

The UV-Vis MS analysis may be an objective index to evaluate the grade of endometrial endometrioid cancer by analyzing the spectral values of ultraviolet and visible light transmittances and the degree of ultraviolet light- absorbing materials influencing nuclear colors in the same nuclear region. We will apply and develop this as an objective index for the diagnosis and prognosis of patients with various cancers.

\section{Acknowledgment}

This study was supported by a Grant for Hirosaki University Institutional Research (2010 2012). The authors are grateful to Drs. Tatsusuke Sato and Terumasa Takamatu of Claro, Inc., for technical advice and expertise on UV-Vis MS.

\section{Conflict of interests}

The authors declare that there is no conflict of interests regarding the publication of this paper.

\section{References}

1. A report of the gynecologic oncology committee of Japan (2008) Acta Obstet Gynecol Jpn 60: 1001-1085.

2. Zeskind BJ, Jordan CD, Timp W, Trapani L, Waller G, et al. (2007) Nucleic acid and protein mass mapping by live-cell deep-ultraviolet microscopy. Nat Methods 4: 567-569.

3. Cheung MC, Evans JG, McKenna B, Ehrlich DJ (2011) Deep ultraviolet mapping of intracellular protein and nucleic acid in femtograms per pixel. Cytometry A 79: 920-932.

4. Yoshioka H, Hoshiai K, Nakamura T, Sato T, Washiya K, et al. (2014) Usefulness of Ultraviolet - Microscopic Spectroscopy on Unstained cells by Liquid-based Cytology for Objective Differentiation between Noncancer from Cancer cells. Hirosaki Med J 65: 82-94.

5. Yoshioka H, Hoshiai K, Nakamura T, Horie K, Washiya K, et al. (2015) Usefulness of Evaluation of Nuclear Color by Visible-Microscopic Spectroscopy for Objective Differentiation between Non-Cancer and Cancer Cells Prepared Using Liquid-Based Cytology. J Cytol Histol 6: 308.

6. Bettinger C, Zimmermann HW (1991) New investigations on hematoxylin, hematein, and hematein-aluminium complexes. I. Spectroscopic and physico-chemical properties of hematoxylin and hematein. Histochemistry 95: 279-288.

7. Gurley AM, Hidvegi DF, Bacus JW, Bacus SS (1990) Comparison of the Papanicolaou and Feulgen staining methods for DNA quantification by image analysis. Cytometry 11: 468-474.

8. Feng C, Shuzhen C, Libo Z (2007) New Abnormal Cervical Cell Detection Method of Multi-Spectral Pap Smears. WUJNS 12: 476-480.

9. Biesterfeld S, Beckers S, Del Carmen Villa Cadenas M, Schramm M (2011) Feulgen staining remains the gold standard for precise DNA image cytometry. Anticancer Res 31: 53-58.

10. Zink D, Fischer AH, Nickerson JA (2004) Nuclear structure in cancer cells. Nat Rev Cancer 4: 677-687. 
Citation: Yoshioka H, Kondo H, Horie K, Washiya K, Watanabe J (2015) Evaluation of Endometrial Endometrioid Carcinoma Grade using Ultraviolet- Visible-Light Microscopic Spectroscopy. J Cytol Histol 6: 381. doi:10.4172/2157-7099.1000381

Page 7 of 7

11. Koss LG (2006) Fundamental concepts of neoplasia: Benign tumor and Cancer. In: Koss LG, Melamed MR: Koss'Diagnostic Cytology. (4thedn), Lippincott Williams \& Wilkins, New York.

12. Koss LG (2006) Morphologic Response of Cells to Injury. In: Koss LG, MR Melamed MR, Koss'Diagnostic Cytology. (4thedn), Lippincott Williams \& Wilkins, New York.

13. Meier H (2008) UV/Vis Spectroscopy. In: Hesse M, Meier H, Zeeh B, Spectroscopic Methods in Organic Chemistry. (2ndedn), Thieme, New York.
14. Lakowicz JR (2009) Fluorophres. In: Lakowicz JR, Principles of Fluorescence Spectroscopy. (3rdedn), Springer, Baltimore.

15. Yanai H (2010) Discriminant analysis. In: Yanai H, Practical Mutivariate Analysis Greate Utilitieson Exel. OMS publshing Inc., Tokyo.

16. Alberts, Johnson A (2008) Basic Genetic Mechanisms. In: Molecular Biology of The Cell. (5thedn), Garland Science, New York. 